\title{
Interações medicamentosas potencias em unidades de terapia intensiva de um hospital do Sul do Brasil
}

\section{Potential drug-drug interactions in intensive care units of a hospital in Southern Brazil}

\author{
Gustavo Henrique Oliveira-Paula ${ }^{1}$; Francieli Pereira ${ }^{2}$; Mayara Ticianelli Paccola ${ }^{3}$; \\ Airton da Cunha Martins-Junior'; Ester Massae Okamoto Dalla-Costa ${ }^{5}$
}

\begin{abstract}
Resumo
As interações medicamentosas são importantes causas de reações adversas em unidades de saúde. O elevado consumo de medicamentos em unidades de terapia intensiva predispõe os pacientes a um risco potencial de ocorrência de interações medicamentosas. O objetivo deste estudo foi determinar a frequência e as características das interações medicamentosas potenciais em unidades de terapia intensiva do Hospital Universitário da Universidade Estadual de LondrinaForam analisadas as prescrições de pacientes maiores de 18 anos internados no período de janeiro a maio de 2010, com permanência de pelo menos quatro dias. A análise das interações medicamentosas foi realizada utilizando-se o sistema Micromedex Drug-Reax ${ }^{\circledR}$. As interações foram classificadas por gravidade, tempo necessário para o início dos efeitos adversos, mecanismo de ação e qualidade da evidência científica. Além disso, foram analisados os possíveis eventos adversos relacionados às interações, bem como as estratégias de manejo e monitoramento recomendadas. Ao todo foram identificadas 198 diferentes interações medicamentosas potenciais com a ocorrência de 1242 episódios. Destes, $43 \%$ foram caracterizados por interações de gravidade moderada, $35 \%$ graves, $16 \%$ leves e $6 \%$ contra-indicadas. A ineficácia terapêutica foi o possível evento adverso mais frequente $(18 \%)$ e a principal estratégia de manejo recomendada foi o ajuste de dose $(35,6 \%)$. As interações mais frequentes foram: fentanil + midazolam $(8,6 \%)$, fenitoína + ranitidina $(5,5 \%)$ e midazolam + ranitidina $(4,8 \%)$. Os resultados identificados demonstram a importância das interações medicamentosas como evento adverso significativo em unidades de terapia intensiva e, portanto, medidas preventivas são necessárias para minimizar este problema.
\end{abstract}

Palavras-chave: Prescrição de medicamentos. Interações de medicamentos. Farmacoepidemiologia.

\begin{abstract}
Drug-drug interactions are important causes of adverse reactions in health units. The high consumption of medicines in intensive care units predisposes patients to potential drug-drug interactions. The aim of this study was to examine the frequency and the characteristics of drug-drug interactions in intensive care units of University Hospital of State University of Londrina. We analyzed the prescriptions of patients over 18 years, admitted from January to May 2010, who remained hospitalized for at least four days. The analysis of drugdrug interactions was carried out using the Micromedex Drug-Reax ${ }^{\circledR}$ system. The interactions were classified
\end{abstract}

\footnotetext{
${ }^{1}$ Doutorando do Programa de Pós-Graduação em Farmacologia da Faculdade de Medicina de Ribeirão Preto da Universidade de São Paulo (FMRP-USP). E-mail: gustavodepaula 88@yahoo.com.br.

${ }^{2}$ Farmacêutica. Mestranda do Programa de Pós-Graduação em Ciências Farmacêuticas da Faculdade de Ciências Farmacêuticas de Ribeirão Preto da Universidade de São Paulo (FCFRP-SP). E-mail: francieli.pereira@usp.br.

${ }^{3}$ Farmacêutica. Mestranda do Programa de Pós-Graduação em Ciências Farmacêuticas pela Universidade Federal do Paraná (UFPR).

${ }^{4}$ Doutorando do programa de Toxicologia da Faculdade de Ciências Farmacêuticas de Ribeirão Preto - USP. E-mail: airton_farma57@ hotmail.com

${ }^{5}$ Doutora em Saúde Pública pela Faculdade de Saúde Pública da Universidade de São Paulo, Brasil. Professora Adjunta da Universidade Estadual de Londrina. E-mail: esterdallacosta@yahoo.com.br
} 
by severity, time required for the onset of adverse effects, mechanism of action and quality of scientific evidence. Moreover, the possible adverse events were analyzed, as well as the recommended strategies of management and monitoring. Altogether, 198 different potential drug-drug interactions were identified with the occurrence of 1242 episodes. Of these, $43 \%$ were characterized by moderate interactions, $35 \%$ major, $16 \%$ minor and $6 \%$ contraindicated. The therapeutic inefficacy was the most frequent possible adverse event $(18 \%)$ and the main recommended strategy of management was the dose adjustment (35.6\%). The most frequent interactions were: fentanyl + midazolam $(8.6 \%)$, phenytoin + ranitidine $(5.5 \%)$ and midazolam + ranitidine (4.8\%). These results demonstrate the importance of drug-drug interactions as a significant adverse event in intensive care units and thus, preventive measures are required to minimize this problem.

Key words: Drug prescriptions. Drug-drug interactions. Pharmacoepidemiology.

\section{Introdução}

A variedade de fármacos que compõe o arsenal terapêutico atual é, sem dúvida, responsável pelo tratamento bem-sucedido de inúmeras enfermidades, muitas das quais até alguns anos, não possuíam tratamento realmente eficaz (FUCHS; WANNMACHER; FERREIRA, 2004). Apesar dos benefícios, essa variedade também levou à administração de fármacos em regime de polifarmácia, podendo levar a ocorrência de interações medicamentosas (IM) - definidas como alterações das respostas farmacológicas ou clínicas decorrentes de uma combinação de fármacos (JANKEL; SPEEDIE, 1990).

A ocorrência de IM está relacionada à idade, sexo, número de medicamentos em uso, duração do tratamento, entre outros fatores (CRUCIOLSOUZA; THOMSON, 2006). Embora em diversas situações a combinação de fármacos tenha finalidade terapêutica (WILTINK, 1998), sabe-se que, de maneira geral, as IM podem levar à toxicidade ou redução dos benefícios terapêuticos, comprometendo assim a segurança do paciente (OBACH, 2003).

No ambiente hospitalar, estudos estimam que entre $5 \%$ a $20 \%$ das reações adversas decorrentes de IM resultam em prolongamento do tempo de internação ou óbito (CLASSEN et al., 1997), além do aumento dos custos hospitalares (ALMEIDA; GAMA; AKAMINE, 2007). De modo particular, em unidades de terapia intensiva (UTI) o risco para a ocorrência de IM é maior, dada a complexidade da farmacoterapia empregada, envolvendo o uso simultâneo de diversos fármacos de diferentes classes terapêuticas (VAN DEN BEMT et al., 2002). Além disso, pacientes em estado crítico são mais suscetíveis às reações adversas provenientes de IM, uma vez que a gravidade do quadro clínico e o comprometimento da função renal e hepática podem afetar a resposta farmacológica aos medicamentos utilizados (ZAGLI et al., 2008).

Uma série de grupos de pesquisa têm avaliado IM potenciais em UTI e a frequência dessas IM tem sido bastante variável (ASKARI et al., 2013; DE CARVALHO et al., 2013; HASAN et al., 2012; MENESES; MONTEIRO, 2000; REIS; CASSIANI, 2011; SIERRA et al., 1997; SMITHBURGER; KANE-GILL; SEYBERT, 2012). Um dos primeiros estudos neste sentido foi realizado na Espanha por Sierra et al. (1997) e constatou que $44,3 \%$ dos pacientes internados em UTI apresentavam IM potenciais em suas prescrições. No Brasil, trabalho realizado por Meneses e Monteiro (2000) verificou uma frequência bem maior, na faixa dos $90 \%$. Mais recentemente, um estudo multicêntrico brasileiro detectou IM potenciais em prescrições de 70,6\% dos pacientes internados em UTI de hospitais universitários (DE CARVALHO et al., 2013).

De modo geral, as IM potenciais podem ser caraterizadas por gravidade, mecanismo de ação e evento adverso esperado (KLASCO; MOORE, 2008) e assim como a frequência, as características das IM em UTI também têm variado nos diferentes estudos. A frequência de IM consideradas graves, por exemplo, está na faixa dos $30 \%$ em estudos 
brasileiros (ALMEIDA; GAMA; AKAMINE, 2007; DE CARVALHO et al., 2013), enquanto um estudo holandês demonstrou a frequência de $11 \%$ para IM desta gravidade (ASKARI et al., 2013).

Diante da variabilidade da frequência e das características das IM potenciais em UTI de diferentes hospitais, novas investigações agregarão conhecimento à epidemiologia das IM em UTI. Além disso, a realização de novos estudos é importante para evidenciar a gravidade deste problema. Deste modo, o presente trabalho teve como objetivo determinar a frequência e as características das IM potenciais em UTI de um hospital do Sul do Brasil.

\section{Metodologia}

\section{Delineamento do estudo}

Trata-se de um estudo transversal e descritivo, que avaliou a frequência e as características das interações medicamentosas potenciais em pacientes internados em três UTI do Hospital Universitário da Universidade Estadual de Londrina (HU/UEL).

\section{Local do estudo, população e amostra}

O presente estudo foi realizado em UTI do HU/ UEL. Este hospital é um órgão suplementar da UEL, que presta serviços de atenção terciária a pacientes de cerca de 250 municípios do Paraná e de mais de 100 cidades de outros estados, de várias regiões do País. Conta com 333 leitos ativados, todos conveniados com o Sistema Único de Saúde (SUS).

Para o estudo, foram analisados os prontuários de pacientes internados no período de janeiro a maio de 2010 em três Unidades de Terapia Intensiva do HU/UEL: UTI I e II, que contam com 10 e 7 leitos, respectivamente; e a UTI da Unidade de Queimados, que possui 6 leitos. Os prontuários foram selecionados de acordo com os seguintes critérios: paciente maior de 18 anos; prescrição de dois ou mais medicamentos nas primeiras 24 horas de internação e período mínimo de permanência na
UTI de quatro dias.

A coleta de dados deu-se em três diferentes momentos do período de internação do paciente na UTI. O primeiro momento foi denominado "prescrição de 24 horas" e refere-se às prescrições coletadas no dia seguinte à admissão do paciente na UTI. No segundo momento optou-se pela avaliação das prescrições do meio da internação, determinando-se a mediana da internação de cada paciente, o que foi denominado "prescrição da mediana da internação". Por fim, no terceiro momento, denominado "prescrições de alta", foram avaliadas as prescrições constantes no último dia do paciente na UTI.

\section{Procedimento para coleta de dados}

Os dados dos prontuários foram registrados em formulário próprio, sendo coletadas as seguintes informações: dados de identificação do paciente, data e motivo de internação, medicamentos prescritos, evolução clínica e resultados de exames laboratoriais.

Os medicamentos prescritos foram posteriormente homogeneizados em sua denominação genérica e classificados de acordo com a Classificação Anatômica-TerapêuticaQuímica, mais conhecida pela sigla em inglês ATC - Anatomical-Therapeutic-Chemical Classification. Essa classificação pode variar de acordo com a finalidade terapêutica do medicamento; porém, no presente trabalho, a classificação teve como base a finalidade terapêutica mais comum pela limitação na obtenção desta informação nos registros analisados.

Apesar de existirem diferentes bases de dados sobre IM, no presente estudo as interações foram analisadas com a utilização do Sistema Micromedex Drug-Reax ${ }^{\circledR}$ (KLASCO; MOORE, 2008), por sua facilidade de acesso, e por ser considerado uma das bases de dados mais completa (ABARCA et al., 2004). O funcionamento do sistema exige a inserção do nome de cada medicamento em sua Denominação Comum Internacional (DCI) ou 
sua denominação genérica em inglês, presente em uma prescrição, a dose e via de administração, fornecendo a informação sobre as IM existentes. $\mathrm{O}$ sistema fornece ainda os seguintes detalhes de cada IM: classificação da gravidade das reações adversas da IM, eventos adversos esperados mais frequentes, tempo previsto para o início das eventos adversos, mecanismo de ação da IM, recomendações e sugestões para monitoramento e manejo clínico da IM, qualidade da evidência em literatura e as referências bibliográficas respectivas.

Embora as IM possam ser classificadas por diferentes critérios, no presente trabalho optouse pela classificação de acordo com os seguintes quesitos: gravidade, tempo previsto para o início dos efeitos, evidência científica e mecanismo de ação (KLASCO; MOORE, 2008).

Com relação à gravidade, as IM foram agrupadas em:

- Contra-indicada: quando a administração concomitante dos fármacos não é recomendada em hipótese alguma;

- Grave: quando apresenta risco de vida ao paciente ou requer intervenção médica para minimizar ou prevenir reações adversas;

- Moderada: quando resulta em exacerbação da condição de saúde do paciente e/ou quando requer mudanças na farmacoterapia;

- Leve: quando resulta em efeitos clínicos limitados, podendo incluir aumento na frequência ou gravidade dos efeitos colaterais, mas que geralmente não requerem mudanças na farmacoterapia.

Quanto ao tempo previsto para o início dos efeitos adversos, a IM foi classificada em: rápida, quando os efeitos adversos previstos poderiam ocorrer imediatamente ou nas primeiras 24 horas após a administração dos medicamentos; tardia, quando os efeitos adversos previstos poderiam ocorrer depois de 24 horas após a administração dos medicamentos.
No que diz respeito à qualidade da evidência em literatura científica, as IM foram classificadas em: excelente, quando há a existência de estudos controlados que estabeleceram claramente a possibilidade da IM; boa, quando há existência de estudos que sugerem fortemente a possibilidade de ocorrência de IM, mas não há estudos bem controlados; regular, quando existem poucos estudos de qualidade para um fármaco que seja da mesma classe terapêutica de um dos envolvidos no par suspeito de IM, ou as considerações farmacológicas do par levam os clínicos a suspeitarem sobre a possibilidade de ocorrência de IM.

Por fim, os mecanismos de ação das IM foram classificados em: farmacodinâmico, quando um fármaco possui a capacidade de alterar a ação farmacológica de outro, sem alterar sua concentração no sítio de ação; farmacocinético, quando um fármaco possui a capacidade de aumentar ou diminuir a concentração de outro em quaisquer das etapas do processo farmacocinético.

\section{Organização e análise dos dados}

A análise dos dados foi realizada por meio da estatística descritiva. Foram calculadas as frequências absolutas e relativas das características clínicas e demográficas dos pacientes, bem como das caraterísticas relacionadas à farmacoterapia. Foram verificadas também as frequências absolutas e relativas das IM e de suas características. Além disso, foram calculadas as médias \pm desvio padrão dos seguintes itens: idade, tempo de internação na UTI e medicamentos por prescrição. A tabulação e análise dos dados foram realizadas com a utilização de planilhas do programa Microsoft Office Excel®.

\section{Aspectos éticos}

O estudo foi desenvolvido respeitando-se todos os princípios éticos constantes na Resolução 196/96 sobre pesquisa envolvendo seres humanos. Não houve intervenção direta com os pacientes, o 
que isentou a necessidade de utilização do termo de consentimento livre e esclarecido. O sigilo e a confidencialidade dos dados coletados foram assegurados. O projeto foi submetido e aprovado pelo Comitê de Ética em Pesquisa da UEL (Parecer CEP/UEL no 262/09). Não há conflito de interesses.

\section{Resultados}

Caracterização demográfica e clínica dos pacientes

Atenderam aos critérios de seleção do estudo 133 pacientes internados nas três UTI do HU/UEL no período referente ao meses de janeiro a maio de 2010, sendo 78 (59\%) do sexo masculino e 55 (41\%) do sexo feminino. A idade dos pacientes variou de 18 a 96 anos, com média de 54,7 $\pm 19,7$ anos. O tempo médio de internação dos pacientes na UTI foi de 14,8 dias, com variação entre 4 a 89 dias e mediana de 10 dias. Com relação ao desfecho da internação, 53 (40\%) pacientes receberam alta e 80 (60\%) foram a óbito.

As causas mais comuns de internação diagnóstico principal registrado no prontuário, foram: queimadura $(21,8 \%)$, choque séptico $(15,8 \%)$, acidente vascular cerebral $(8,3 \%)$, acidente automobilístico $(3,8 \%)$ e pneumonia $(3,8 \%)$.

Embora 42 (32\%) pacientes não fossem portadores de nenhuma comorbidade, verificouse que 37 (28\%) dos pacientes possuíam uma comorbidade, 27 (20\%) dos pacientes possuíam duas, $23(17 \%)$ possuíam três e $4(3 \%)$ pacientes possuíam quatro comorbidades. As comorbidades mais prevalentes foram a hipertensão arterial, que estava presente em $50(29,6 \%)$ pacientes e o diabetes em $19(11,2 \%)$ pacientes.

\section{Perfil da farmacoterapia}

Com relação à prescrição, um total de 155 diferentes medicamentos foram prescritos no período analisado, com uma média de $10 \pm 4$ medicamentos por prescrição. A ocasião em que um mesmo medicamento foi prescrito mais de uma vez ocorreu inúmeras vezes, o que totalizou a prescrição de 4079 medicamentos no período.

Os medicamentos mais prevalentes foram: heparina, presente em prescrições de 106 $(79,7 \%)$ pacientes; ranitidina em 90 (67,7\%); metoclopramida em 88 (66,2\%); omeprazol em 73 (54,9\%); bromoprida em $70(52,6 \%)$.

De acordo com a classificação ATC dos medicamentos, considerados os dois primeiros níveis, as classes farmacoterapêuticas mais prescritas foram: antimicrobianos de uso sistêmico $(\mathrm{N} 01=356$; 23\%); antiespasmódicos e anticolinérgicos $(\mathrm{A} 03=166 ; 9,6 \%)$; antiácidos gástricos $(\mathrm{A} 02=164$; $11 \%)$; medicamentos usados na terapia cardíaca $(\mathrm{C} 01=155 ; 10 \%)$ e analgésicos (N02=138; 9\%).

Com relação às vias de administração dos medicamentos, observou-se que a maioria dos medicamentos foi prescrita para administração por via endovenosa $(\mathrm{n}=2.835 ; 69,5 \%)$, seguida da sonda enteral $(n=429 ; 10,5 \%)$ e via subcutânea $(n=333$; $8,2 \%)$.

Tabela 1 - Distribuição das dez IM potenciais mais frequentes em prescrições de pacientes internados em unidades de terapia intensiva do Hospital Universitário da Universidade Estadual de Londrina.

\begin{tabular}{cccc}
\hline Interação Medicamentosa & Gravidade & $\mathbf{N}^{\star}$ & $\mathbf{\%}$ \\
\hline Fentanil + Midazolam & Grave & 107 & 8,6 \\
Fenitoína + Ranitidina & Leve & 68 & 5,5 \\
Midazolam + Ranitidina & Moderada & 59 & 4,8 \\
Midazolam + Omeprazol & Moderada & 50 & 4,0 \\
Fenitoina + Omeprazol & Moderada & 34 & 2,7 \\
AAS + Heparina & Grave & 33 & 2,7 \\
Fentanil + Linezolida & Grave & 27 & 2,2 \\
Linezolida + Metoclopramida & Grave & 26 & 2,1 \\
Fenitoina + Midazolam & Moderada & 25 & 2,0 \\
Dexametasona + Fenitoina & Moderada & 25 & 2,0
\end{tabular}

AAS: ácido acetilsalicílico

${ }^{*}$ Refere-se ao número de episódios de IM potenciais.

Fonte: Autores.

\section{Interações medicamentosas potenciais: identificação e caracterização}

Entre os 133 pacientes, 99 (74\%) deles tiveram pelo menos uma IM em suas prescrições de 24 horas, 
107 (80\%) pacientes na prescrição da mediana da internação e 99 (74\%) pacientes na prescrição da alta. Na média dos três períodos analisados, 76\% dos pacientes apresentaram pelo menos uma IM em suas prescrições.

Ao todo, foram encontradas 198 diferentes combinações de medicamentos com potencial de IM. Essas combinações se repetiram, totalizando 1242 episódios potenciais de IM. Quando os episódios de IM foram estratificados por momento de internação, constatou-se a presença de 371 (29,9\%) episódios nas prescrições de 24 horas, 434 $(34,9 \%)$ nas prescrições da mediana e $437(35,2 \%)$ nas prescrições de alta.

As IM mais frequentes no presente estudo, foram: fentanil + midazolam $(8,6 \%)$, seguida de fenitoína + ranitidina $(5,5 \%)$ e midazolam + ranitidina $(4,8 \%)$, como pode ser verificado na Tabela 1 , que apresenta ainda a classificação das interações de acordo com a gravidade da interação.

As IM foram classificadas de acordo com Micromedex Drug-Reax ${ }^{\circledR}$ em relação a gravidade, tempo de início dos efeitos, qualidade da evidência científica e mecanismo de ação. Do total de 1242 episódios de IM, 538 (43\%) foram caracterizados por IM de gravidade moderada, 437 (35\%) por IM graves, 194 (16\%) por IM leves e 73 (6\%) por IM contra-indicadas (Figura 1A). Com relação ao tempo de início dos efeitos, 532 (42,8\%) episódios foram compostos por IM de efeito tardio, enquanto 283 $(22,8 \%)$ foram compostos por IM de efeito rápido; em $427(34,4 \%)$ episódios o início dos efeitos da IM foi classificado como desconhecido (Figura 1B). As IM com evidência científica de qualidade classificada como boa foram responsáveis por 583 $(46,9 \%)$ episódios, ao passo que as de qualidade regular foram responsáveis por $458 \quad(36,9 \%)$ episódios e as de excelente qualidade, 201 (16,2\%) (Figura 1C). As IM cujo mecanismo de ação é descrito como farmacocinético fizeram parte de 717 $(57,7 \%)$ episódios, enquanto as IM com mecanismo farmacodinâmico foram responsáveis por 369
(29,7\%); já os episódios em que as IM tinham mecanismo de ação desconhecido totalizaram 156 $(12,6 \%)$ (Figura 1D).

Figura 1 - Distribuição das IM classificadas segundo gravidade (A), tempo de início dos efeitos (B), qualidade da evidência científica (C) e mecanismo de ação (D).
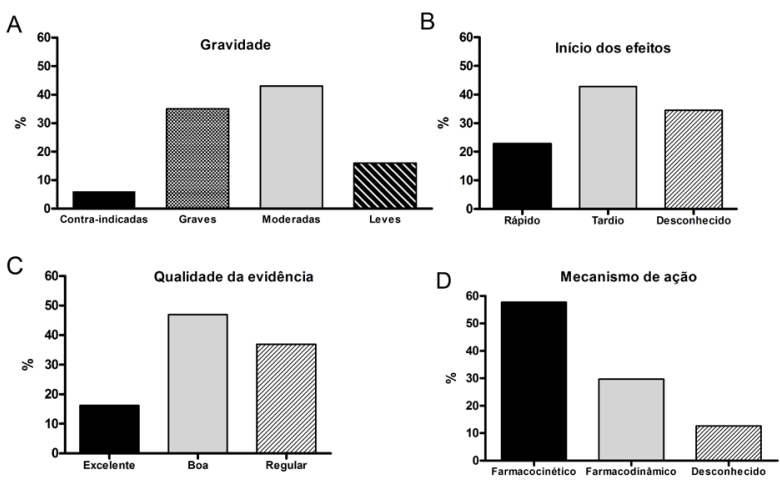

Fonte: Autores.

As porcentagens apresentadas são referentes ao número de episódios de IM em que as variáveis foram constatadas em relação ao número total de episódios (1242).

Em relação aos eventos adversos possíveis de serem induzidos pelas IM potenciais identificadas, a ineficácia terapêutica foi a mais frequente $(18,0 \%)$, seguida de depressão respiratória $(16,6 \%)$ e alteração das concentrações plasmáticas $(13,4 \%)$, como pode ser observado na Tabela 2.

Tabela 2 - Distribuição dos eventos adversos a medicamentos que podem resultar das interações medicamentosas potenciais detectadas no presente estudo.

\begin{tabular}{ccc}
\hline Evento adverso & $\mathbf{N}^{*}$ & $\%$ \\
\hline Inefićácia terapêutica & 223 & 18,0 \\
Depressão Respiratória & 206 & 16,6 \\
Alteração das concentrações plasmáticas & 166 & 13,4 \\
Depressão do SNC & 152 & 12,1 \\
Exacerbação do efeito do medicamento & 115 & 9,3 \\
Hemorragia & 56 & 4,5 \\
Cardiotoxicidade & 55 & 4,4 \\
Ataxia e Hiperreflexia & 35 & 2,8 \\
Síndrome serotoninérgica & 31 & 2,5 \\
Sedação excessiva & 28 & 2,3 \\
Hipocalemia & 23 & 1,9 \\
Miopatia & 21 & 1,7 \\
Hipotensão & 20 & 1,6 \\
Hipercalemia & 20 & 1,6 \\
Hipoglicemia & 14 & 1,1 \\
Reações extrapiramidais & 13 & 1,0 \\
Outros & 64 & 5,2 \\
Total & 1242 & 100 \\
\hline
\end{tabular}

SNC: sistema nervoso central

* Refere-se ao número de episódios de IM em que as variáveis foram constatadas.

Fonte: Autores. 
As estratégias de manejo e monitoramento são apresentadas na Tabela 3. O ajuste de dose foi o manejo mais comum no período analisado, com uma frequência de $35,6 \%$, seguido do monitoramento dos sinais e sintomas $(25,8 \%)$ e da observação da resposta terapêutica $(12,0 \%)$. Quanto às estratégias de monitoramento dos eventos adversos que podem resultar das IM, observou-se que as mais frequentes foram: o monitoramento da função respiratória (14,3\%), monitoramento das concentrações plasmáticas $(13,8 \%)$ e monitoramento do nível de sedação $(7,6 \%)$.

Tabela 3 - Distribuição das estratégias de manejo e monitoramento das IM detectadas no presente estudo.

\begin{tabular}{ccc}
\hline Estratégia & $\mathrm{N}^{*}$ & $\%$ \\
\hline Manejo & & \\
Ajuste de dose & 442 & 35,6 \\
Monitorar sinais e sintomas & 319 & 25,8 \\
Observação da resposta terapêutica & 149 & 12,0 \\
Usar com precaução & 137 & 11,0 \\
Evitar uso conjunto & 101 & 8,1 \\
Outros & 55 & 4,4 \\
Total & 39 & 3,1 \\
Mubstituir suspender um dos medicamentos & 1242 & 100 \\
Monitoramento & 178 & 14,3 \\
Função respiratória & 171 & 13,8 \\
Concentraçós plasmáticas & 95 & 7,6 \\
Nivel de sedação & 70 & 5,6 \\
Pressão arterial & 35 & 2,8 \\
Tempo de protrombina & 35 & 2,8 \\
Potássio sérico & 29 & 2,3 \\
Função Cardiaca & 23 & 1,9 \\
Glicemia & 17 & 1,4 \\
Colesterol e Triglicerídeos & 526 & 42,4 \\
Não informado & 241 & 19,4 \\
Outros & 1242 & 100 \\
Total & & \\
\hline
\end{tabular}

"Refere-se ao número de episódios de IM em que as variáveis foram constatadas.

Fonte: Autores.

\section{Discussão}

Os resultados obtidos demonstram que as IM potenciais são frequentes em UTI, atingindo cerca de $76 \%$ dos pacientes incluídos no estudo. Em estudos realizados em diferentes serviços de saúde, a frequência de IM em UTI variou entre $11 \%$ a $90 \%$ (ASKARI et al., 2013; DE CARVALHO et al., 2013; HASAN et al., 2012; MENESES; MONTEIRO, 2000; REIS; CASSIANI, 2011; SIERRA et al., 1997; SMITHBURGER; KANE-GILL; SEYBERT, 2012). Essa variação pode ser explicada pelas diferentes casuísticas, pelos diferentes perfis das UTI estudadas, bem como pelas variações de sensibilidade e especificidade dos softwares empregados na identificação de IM, além do perfil da farmacoterapia. Porém, é interessante notar que a frequência de IM encontrada nas UTI do HU/UEL foi próxima à de estudos brasileiros desenvolvidos em UTI de diferentes hospitais universitários, com casuísticas e métodos empregados semelhantes (DE CARVALHO et al., 2013; REIS; CASSIANI, 2011). Portanto, o presente estudo reforça a alta frequência de IM em UTI de hospitais universitários brasileiros. Essa alta frequência é preocupante, não apenas em decorrência das possíveis consequências, mas também porque os hospitais universitários, pelo seu caráter de ensino, devem servir de exemplo para demais unidades e aos profissionais da saúde (MEDICI, 2001).

Embora no presente estudo as IM com mecanismo de ação farmacocinético tenham prevalecido (Figura 1D), a interação entre fentanil e midazolam, cujo mecanismo de ação é farmacodinâmico, foi a mais comum nas prescrições avaliadas (Tabela 1), tal como em trabalhos brasileiros de casuística semelhante (DE CARVALHO et al., 2013; REIS; CASSIANI, 2011). Esta IM fundamenta-se no sinergismo farmacológico com o objetivo de produzir sedação por meio da utilização de um analgésico opióide (fentanil) associado a um benzodiazepínico (midazolam). Em UTI esta IM é amplamente empregada com finalidade terapêutica, pois busca oferecer conforto ao paciente crítico em ventilação mecânica, alívio da dor e da ansiedade, sincronia do paciente com o ventilador e otimizar a oxigenação (DEVLIN; ROBERTS, 2009).

Apesar da finalidade terapêutica, a IM fentanil + midazolam é considerada grave, pois pode resultar em depressão respiratória e sedação excessiva (RICHMAN et al., 2006). Não foi localizada na literatura consultada uma especificação relativa ao tempo de início dos efeitos adversos relacionados a essa interação, mas recomenda-se que o paciente seja monitorado constantemente. Além disso, sugerese a redução da dose de um dos medicamentos, ou de ambos, para que os efeitos adversos sejam minimizados (KLASCO; MOORE, 2008).

Comparativamente, a distribuição das frequências 
de gravidade das IM do presente estudo mostrou-se semelhante à de outros estudos que avaliaram IM em UTI (ALMEIDA; GAMA; AKAMINE, 2007; DE CARVALHO et al., 2013). Embora a maioria dos episódios de IM detectados neste estudo tenha sido de gravidade moderada, percebe-se que o número de IM graves somados ao número de IM contra-indicadas merece destaque (Figura 1A). Além disso, considerando-se que os pacientes de UTI frequentemente têm idade avançada e alterações fisiológicas importantes, somando-se a condições clínicas desfavoráveis ao metabolismo dos fármacos, como choque, insuficiência renal e hepatopatias, podese supor que a identificação de uma IM, até mesmo as menos graves, tenha importância na prevenção de efeitos adversos indesejáveis (HAMMES et al., 2008).

Quanto aos eventos adversos possíveis de serem induzidos por IM, ineficácia terapêutica foi o mais frequente deles(Tabela3). Um dos principais fármacos que predispõe à IM desencadeadoras desse possível evento adverso é a fenitoína (DE CARVALHO et al., 2013). Este fármaco é um potente indutor enzimático, e assim, sua interação com outros fármacos, como por exemplo, a nifedipina e o midazolam, pode acarretar na redução das concentrações plasmáticas dos últimos, levando à ineficácia terapêutica (DE CARVALHO et al., 2013). De fato, a fenitoína esteve presente em diversos episódios de IM deste estudo (dados não mostrados), o que possivelmente explica a alta frequência do evento adverso supracitado. Vale salientar que ineficácia terapêutica acarreta em aumento da morbidade e mortalidade, redução da qualidade de vida e aumento dos custos hospitalares (DIAS et al., 2011).

O segundo possível evento adverso decorrente de IM mais comum foi depressão respiratória (Tabela 3), que pode ser consequência da alta frequência da IM fentanil + midazolam, bem como da soma de outras IM que levam a este efeito, como, por exemplo, fenobarbital + morfina, fentanil + tiopental e fentanil + lorazepam. A depressão respiratória caracterizase pela redução da efetividade da ventilação do paciente e pode levar ao aumento da morbidade e mortalidade (DAHAN et al., 2013). Um dos meios de se evitar a ocorrência desse evento adverso é ajustar a dose de pelo menos um dos medicamentos interagentes (KLASCO; MOORE, 2008), o que explica a alta frequência desta estratégia de manejo no presente estudo (Tabela 3). Adicionalmente, várias estratégias de monitoramento visando evitar a depressão respiratória são recomendadas, como avaliação do nível de sedação e da função respiratória (HAGLE et al., 2004). De fato, monitorar a função respiratória foi a estratégia de monitoramento mais recomendada para as IM do presente estudo (Tabela 3), possivelmente em decorrência da alta frequência de IM que potencialmente levam à depressão respiratória.

Finalmente, como esperado, o monitoramento dos sinais e sintomas foi outra estratégia de manejo frequentemente recomendada (Tabela 3 ), o que pode estar ligado ao fato de a maioria das IM detectadas no presente estudo caracterizar-se por levar a ocorrência de eventos adversos tardios (Figura 1B).

Tendo em vista o impacto negativo das IM em UTI, medidas de prevenção fazem-se necessárias. Uma medida que tem se mostrado eficaz é o emprego de sistemas informatizados capazes de detectar e prevenir a ocorrência de IM, agregando assim maior segurança à prescrição médica e ao paciente (SMITHBURGER; KANE-GILL; SEYBERT, 2012). A promoção da atenção multiprofissional ao paciente é outra importante medida que pode prevenir este problema. Particularmente, a participação do farmacêutico no acompanhamento das prescrições médicas tem se mostrado capaz de reduzir a frequência de IM em UTI (HASAN et al., 2012). Desse modo, ressalta-se a importância desse profissional no acompanhamento do consumo, do uso racional e na prevenção da ocorrência de eventos adversos relacionados a medicamentos, principalmente em UTI onde a utilização de medicamentos é elevada e o estado de saúde dos pacientes é crítico.

O presente estudo apresenta algumas limitações. 
Por se tratar de um estudo retrospectivo, somado ao fato dos registros em prontuário muitas vezes se apresentarem incompletos, não foi possível avaliar se os eventos adversos decorrentes das IM realmente ocorreram e, portanto, novos estudos de caráter prospectivo são necessários para esclarecer esta questão. Outra limitação que deve ser salientada é o fato de alguns fármacos não serem cadastradas no sistema Micromedex Drug-Reax ${ }^{\circledR}$ - o que pode levar à não identificação das possíveis IM desencadeadas por esses fármacos. Portanto, a frequência de IM em UTI pode ter sido subestimada no presente estudo. Ainda assim, é inegável que os resultados apresentados neste estudo representam um importante sinal de alerta para o problema das IM em UTI.

\section{Conclusão}

Constatou-se uma alta frequência de IM potenciais nas prescrições de pacientes internados em UTI no período compreendido entre janeiro e maio de 2010. Embora o número de episódios de IM moderadas tenha prevalecido, observou-se um grande número de episódios de IM graves. Estes dados demonstram a relevância das IM como evento adverso na UTI, que podem produzir impactos negativos, tanto de ordem econômica quanto clínica.

O reconhecimento da IM pode ser realizado no momento da prescrição, da dispensação e na administração dos medicamentos sendo, portanto, recomendada maior valorização desta possibilidade e o estabelecimento de estratégias de intervenção. Destaca-se a importância da utilização de sistemas informatizados e da promoção da atenção multiprofissional ao paciente, a fim de reduzir a ocorrência desse evento adverso.

\section{Agradecimentos}

À Pró-Reitoria de Pesquisa e Pós-Graduação da Universidade Estadual de Londrina pela bolsa de iniciação científica concedida e à direção e equipe do HU/UEL pela permissão de acesso aos dados.

\section{Referências}

ABARCA, J.; MALONE, D. C.; ARMSTRONG, E. P.; GRIZZLE, A. J.; HANSTEN, P. D.; VAN BERGEN, R. C.; LIPTON, R. B. Concordance of severity ratings provided in four drug interaction compendia. Journal of the American Pharmacists Association, Washington, v. 44, n. 2, p. 136-141, 2004.

ALMEIDA, S.; GAMA, C. S.; AKAMINE, N. Prevalência e classificação de interações entre medicamentos dispensados para pacientes em terapia intensiva. Einstein, São Paulo, v. 5, n. 4, p. 347-351, 2007.

ASKARI, M.; ESLAMI, S.; LOUWS, M.; WIERENGA, P.C.; DONGELMANS, D. A.; KUIPER, R. A.; ABU-HANNA, A. Frequency and nature of drug-drug interactions in the intensive care unit. Pharmacoepidemiology and Drug Safety, Bethesda, v. 22, n. 4, p. 430-437, 2013.

CLASSEN, D. C.; PESTOTNIK, S. L.; EVANS, R. S.; LLOYD, J. F.; BURKE, J. P. Adverse drug events in hospitalized patients. Excess length of stay, extra costs, and attributable mortality. JAMA, Chicago, v. 277, n. 4, p. 301-306, 1997.

CRUCIOL-SOUZA, J. M.; THOMSON, J. C. Prevalence of potential drug-drug interactions and its associated factors in a Brazilian teaching hospital. Journal of Pharmaceutical Sciences, Washington, v. 9, n. 3, p. 427-433, 2006.

DAHAN, A.; OVERDYK, F.; SMITH, T.; AARTS, L.; NIESTERS, M. Pharmacovigilance: a review of opioidinduced respiratory depression in chronic pain patients. Pain Physician, Paducah, v. 16, n. 2, p. E85-94, 2013.

DE CARVALHO, R. E. F. L.; REIS, A. M. M.; DE FARIA, L. M. P.; DE AZEVEDO ZAGO, K. S.; CASSIANI, S. H. D. B. Prevalência de interações medicamentosas em unidades de terapia intensiva no Brasil. Acta Paulista de Enfermagem, São Paulo, v. 26, n. 2, p. 150-157, 2013.

DEVLIN, J. W.; ROBERTS, R. J. Pharmacology of commonly used analgesics and sedatives in the ICU: benzodiazepines, propofol, and opioids. Critical Care Clinics, Philadelphia, v. 25, n. 3, p. 431-449, 2009. 
DIAS, A. M.; CUNHA, M.; SANTOS, A. M. M. D.; NEVES, A. P. G.; PINTO, A. F. C.; SILVA, A. S. A.; CASTRO, S. A. Adesão ao regime Terapêutico na Doença Crónica: Revisão da Literatura. Milleniun, Viseu, v. 40, p. 201-219, 2011.

FUCHS, F. D.; WANNMACHER, L.; FERREIRA, M. B. C. Farmacologia Clínica: fundamentos da terapêtica racional. 3. ed. Rio de Janeiro: Guanabara Koogan, 2004.

HAGLE, M. E.; LEHR, V. T.; BRUBAKKEN, K.; SHIPPEE,A. Respiratory depression in adult patients with intravenous patient-controlled analgesia. Orthopaedic Nursing, Chicago, v. 23, n. 1, p. 18-27, 2004.

HAMMES, J. A.; PFUETZENREITER, F.; DA SILVEIRA, F.; KOENING, A.; WESTPHAL, G. Prevalência de potenciais interações medicamentosas droga-droga em unidades de terapia intensiva. Revista Brasileira de Terapia Intensiva, São Paulo, v. 20, n. 4, p. 349-354, 2008.

HASAN, S. S.; LIM, K. N.; ANWAR, M.; SATHVIK, B. S.; AHMADI, K.; YUAN, A. W.; KAMARUNNESA, M.A. Impact of pharmacists' intervention on identification and management of drug-drug interactions in an intensive care setting. Singapore Medical Journal, Singapura, v. 53, n. 8, p. 526-531, 2012.

JANKEL, C. A.; SPEEDIE, S. M. Detecting drug interactions: a review of the literature. Drug Intelligence and Clinical Pharmacy, Thousand Oaks, v. 24, n. 10, p. 982-989, 1990.

KLASCO, R. K.; MOORE, R. E. Drug Reax ${ }^{\mathbb{R}}$. Greenwold Village (CO): Micromedex 2008.

MEDICI,A.C.Hospitais universitários: passado, presente e futuro. Revista da Associação Médica Brasileira, São Paulo, v. 47, n. 2, p. 149-156, 2001.

MENESES, A. D.; MONTEIRO, H. S. Prevalencia de interaçöes medicamentosas droga-droga" potenciais em duas UTIs (publica X privada) de Fortaleza, Brasil. Revista Brasileira de Teapia Intensiva, São Paulo, v. 12, n. 1, p. 4-7, 2000.

OBACH, R. S. Drug-drug interactions: an important negative attribute in drugs. Drugs of Today, Barcelona, v. 39, n. 5, p. 301-338, 2003.
REIS, A. M.; CASSIANI, S. H. Prevalence of potential drug interactions in patients in an intensive care unit of a university hospital in Brazil. Clinics, São Paulo, v. 66, n. 1, p. 9-15 2011.

RICHMAN, P. S.; BARAM, D.; VARELA, M.; GLASS, P. S. Sedation during mechanical ventilation: a trial of benzodiazepine and opiate in combination. Critical Care Medicine, Mount Prospect, v. 34, n. 5, p. 1395-1401, 2006.

SIERRA, P.; CASTILLO, J.; GOMEZ, M.; SORRIBES, V.; MONTERDE, J.; CASTAÑO, J. Interacciones farmacologicas potenciales y reales en pacientes en estado critico. Revista Española de Anestesiología y Reanimación, Madrid, v. 44, n. 10, p. 383-387, 1997.

SMITHBURGER, P.L.; KANE-GILL, S.L.; SEYBERT, A. L. Drug-drug interactions in the medical intensive care unit: an assessment of frequency, severity and the medications involved. International Journal of Pharmacy Practice, London, v. 20, n. 6, p. 402-408, 2012.

VAN DEN BEMT, P. M.; FIJN, R.; VAN DER VOORT, P. H.; GOSSEN, A. A.; EGBERTS, T. C.; BROUWERS, J. R. Frequency and determinants of drug administration errors in the intensive care unit. Critical Care Medicine, Mount Prospect, v. 30, n. 4, p. 846-850, 2002.

WILTINK, E. H. Medication control in hospitals: a practical approach to the problem of drug-drug interactions. Pharmacy World and Science, Buitenpost, v. 20, n. 4, p. 173-177, 1998.

ZAGLI, G.; TARANTINI, F.; BONIZZOLI, M.; DI FILIPPO, A.; PERIS, A.; DE GAUDIO, A. R.; GEPPETTI,P.Altered pharmacology in the Intensive Care Unit patient. Fundamental and Clinical Pharmacology, Sophia Antipolis, v. 22, n. 5, p. 493-501, 2008.

Recebido em: 7 out. 2013 Aceito em: 14 jul 2014. 